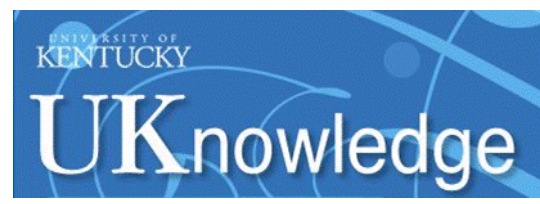

University of Kentucky

UKnowledge

$11-14-2016$

\title{
The Quenching Timescale and Quenching Rate of Galaxies
}

Jianhui Lian

University of Kentucky

Renbin Yan

University of Kentucky, yanrenbin@uky.edu

Kai Zhang

University of Kentucky

Xu Kong

University of Science and Technology of China, China

Follow this and additional works at: https://uknowledge.uky.edu/physastron_facpub

Part of the Astrophysics and Astronomy Commons, and the Physics Commons

Right click to open a feedback form in a new tab to let us know how this document benefits you.

\section{Repository Citation}

Lian, Jianhui; Yan, Renbin; Zhang, Kai; and Kong, Xu, "The Quenching Timescale and Quenching Rate of Galaxies" (2016). Physics and Astronomy Faculty Publications. 466.

https://uknowledge.uky.edu/physastron_facpub/466

This Article is brought to you for free and open access by the Physics and Astronomy at UKnowledge. It has been accepted for inclusion in Physics and Astronomy Faculty Publications by an authorized administrator of UKnowledge. For more information, please contact UKnowledge@lsv.uky.edu. 


\section{The Quenching Timescale and Quenching Rate of Galaxies}

Digital Object Identifier (DOI)

https://doi.org/10.3847/0004-637X/832/1/29

\section{Notes/Citation Information}

Published in The Astrophysical Journal, v. 832, no. 1, 29, p. 1-9.

(c) 2016. The American Astronomical Society. All rights reserved.

The copyright holder has granted the permission for posting the article here. 


\title{
THE QUENCHING TIMESCALE AND QUENCHING RATE OF GALAXIES
}

\author{
Jianhui Lian $^{1,2}$, Renbin YAN $^{2}$, Kai Zhang ${ }^{2}$, And Xu Kong ${ }^{1}$ \\ ${ }^{1}$ CAS Key Laboratory for Research in Galaxies and Cosmology, Department of Astronomy, University of Science and Technology of China, Hefei, Anhui 230026, \\ China; ljhhw@mail.ustc.edu.cn, xkong@ustc.edu.cn \\ ${ }^{2}$ Department of Physics and Astronomy, University of Kentucky, Lexington, Kentucky 40506, USA; renbin@pa.uky.edu \\ Received 2016 June 21; revised 2016 September 2; accepted 2016 September 7; published 2016 November 14
}

\begin{abstract}
The average star formation rate (SFR) in galaxies has been declining since the redshift of 2 . A fraction of galaxies quench and become quiescent. We constrain two key properties of the quenching process: the quenching timescale and the quenching rate among galaxies. We achieve this by analyzing the galaxy number density profile in NUV $-u$ color space and the distribution in NUV $-u$ versus $u-i$ color-color diagram with a simple toy-model framework. We focus on galaxies in three mass bins between $10^{10}$ and $10^{10.6} M_{\odot}$. In the NUV $-u$ versus $u-i$ color-color diagram, the red $u-i$ galaxies exhibit a different slope from the slope traced by the star-forming galaxies. This angled distribution and the number density profile of galaxies in NUV $-u$ space strongly suggest that the decline of the SFR in galaxies has to accelerate before they turn quiescent. We model this color-color distribution with a two-phase exponential decline star formation history. The models with an e-folding time in the second phase (the quenching phase) of $0.5 \mathrm{Gyr}$ best fit the data. We further use the NUV $-u$ number density profile to constrain the quenching rate among star-forming galaxies as a function of mass. Adopting an e-folding time of $0.5 \mathrm{Gyr}$ in the second phase (or the quenching phase), we found the quenching rate to be $19 \% / \mathrm{Gyr}, 25 \% / \mathrm{Gyr}$ and $33 \% /$ Gyr for the three mass bins. These are upper limits of the quenching rate as the transition zone could also be populated by rejuvenated red-sequence galaxies.
\end{abstract}

Key words: galaxies: evolution - galaxies: photometry - galaxies: star formation

\section{INTRODUCTION}

In many large-scale surveys, the bimodal distribution of the galaxy population has been found in color-magnitude and color-mass diagrams (e.g., Strateva et al. 2001; Baldry et al. 2004). In these diagrams, galaxies located between the blue and red populations were often called "green valley galaxies." In addition to colors, many other properties of the green valley galaxies also exhibit intermediate value between the two main populations, such as spectral indices (Kauffmann et al. 2003) and morphological parameters (Driver et al. 2006; Pan et al. 2013). Therefore, the green valley galaxies were considered to represent the transition population from the blue star-forming to red-sequence galaxies (Bell et al. 2004; Faber et al. 2007; Mendez et al. 2011; Gonçalves et al. 2012). However, some studies reported that dusty star-forming galaxies may also exhibit intermediate colors and potentially contaminate the transition population (Brammer et al. 2009; Salim et al. 2009). In addition, by differentiating the morphology of green valley galaxies based on the Galaxy Zoo project, Schawinski et al. (2014) and Smethurst et al. (2015) argued multiple evolution pathways of galaxies through the green valley zone, with early-type galaxies quenching and late-type galaxies stalling. That conclusion could be sensitive to the exact definition (choice of color and range) of the green valley, and is made under the assumption of unchanging morphology. It is also possible that some of the green valley galaxies may come from the red sequence due to rejuvenated star formation. But we expect this fraction to be small (Fang et al. 2012) and we expect galaxies going from the green valley to the blue cloud to be even rarer, although not impossible.

Studies about the evolution of the luminosity (or stellar mass) function of different galaxy types with redshift, support this transition scenario. From $z=1-0$, the number density of red galaxies has increased significantly by a factor of 2 or more
(Blanton 2006; Brown et al. 2007; Faber et al. 2007), while that of blue galaxies decreased slightly or barely changed (Faber et al. 2007; Ramos et al. 2011; Moustakas et al. 2013). This differential evolution of the luminosity function with galaxy types suggests that many blue galaxies have ceased their star formation and evolved into the red sequence during this time period. The rarity of the green valley population implies that the transition timescale must be short (Faber et al. 2007; Martin et al. 2007; Balogh et al. 2011). For example, post-starburst galaxies were considered to be one of the possible candidates of galaxies which are transiting from the blue to red population (Yang et al. 2008; Wong et al. 2012). Their spectra show strong Balmer absorption lines, but negligible [O II] or $\mathrm{H} \alpha$ emission lines, suggesting a violent shutdown of star formation in the recent past with a large amount of A stars still present. However, the small fraction of post-starburst galaxies means they can only account for a small fraction of the increase in the number density profile of red galaxies. The majority of galaxies may have ceased their star formation more smoothly. Before turning quiescent, the star formation rate (SFR) in galaxies has been gradually declining since the redshift of 2 (Madau \& Dickinson 2014). This gradual decline in the SFR introduces the cosmic evolution of main-sequence relation (i.e., mass-SFR relation Daddi et al. 2007; Elbaz et al. 2007; Noeske et al. 2007b).

In this work, concerning the quenching process, the problems we would like to address are: (1) How fast does the galaxy SFR have to decline to turn quiescent? Does it have to be much faster than the long-term decline? (2) What is the quenching rate (the fraction of quenching in a given period) among galaxies? For the evolution speed during quenching, Wetzel et al. $(2012,2013)$ examined the star formation histories of local satellite galaxies and obtained a short quenching e-folding time $(<0.8 \mathrm{Gyr})$ using a cosmological $N$-body 
simulation. Based on the EAGLE cosmological hydrodynamical simulation, Trayford et al. (2016) found the timescale to cross the green valley to be less than 2 Gyr. Recently, based on the Galaxy Evolution Explorer (GALEX) and Sloan Digital Sky Survey (SDSS) data, Schawinski et al. (2014) investigated the green valley galaxies in NUV $-u$ versus $u-i$ color-color diagram and found that early-type green valley galaxies evolve much faster than the late-type ones, with a shorter e-folding time. However, the analysis based on morphology-separated populations may introduce bias in the result, because the galaxy morphology may change significantly along with the transition from blue to red galaxies. In this work, we revisit the UVoptical color-color diagrams to answer how fast the SFR has to decline when a galaxy evolves through the transition (or green valley) zone (Section 3 ).

In terms of the quenching rate, Moustakas et al. (2013) obtained it as a function of stellar mass and redshift by comparing the stellar mass function (SMF) of star-forming and passive galaxies at different redshift. They measured the SMF from $z=0-1$ based on a large galaxy sample from the PRism MUlti-object Survey for intermediate- $z$ and SDSS for low-z. However, the quenching rate determined in this way relies on how well the SMF is measured, which may be limited by sample variance and observation accuracy at high redshift. Also, systematical errors may be hard to avoid, although they did really careful work to address it, when performing a comparison between the two large surveys. Using simply divided star-forming and passive populations, the information of the transition population in between is neglected. In this work, we go beyond simple SFR/color cuts to examine the nature of full color distribution. Throughout this paper, we adopted the cosmological parameters with $H_{0}=70 \mathrm{~km} \mathrm{~s}^{-1} \mathrm{Mpc}^{-1}$, $\Omega_{\Lambda}=0.73$ and $\Omega_{\mathrm{m}}=0.27$. All magnitudes, in this paper, are given in the $\mathrm{AB}$ photometric system.

\section{DATA}

While the optical light of a galaxy is dominated by intermediate- and low-mass stars, its ultraviolet (UV) light typically traces the emission from young massive stars. Therefore, the UV observations provide a good probe of the recent SFR. Combining the UV and optical observations, we are able to investigate the rate at which the specific star formation rates (SSFR) decrease when galaxies evolve through the transition zone. GALEX (Martin et al. 2005) mapped more than half of the sky at far-UV (FUV) and near-UV (NUV), which enables us to study the SFR decline in green valley galaxies with a large and complete sample. The SDSS imaging survey mapped 14,000 square degrees of the sky in five broad optical bands: $u, g, r, i$, and $z$. The depth of the imaging survey reaches $21.3 \mathrm{mag}$ at $i$ band. The SDSS spectroscopic survey obtained optical spectra for galaxies brighter than $17.7 \mathrm{mag}$ in $r$ band with a sky coverage of $\sim 8000$ square degrees. Utilizing the GALEX and SDSS imaging surveys, based on a consistent and unified analysis of galaxies, Blanton et al. (2011) created the NASA-Sloan Atlas (NSA), which includes both the photometry catalog and multiband images from UV to nearIR. In the photometry catalog, Blanton et al. (2011) applied a better treatment of sky subtraction to obtain more accurate photometry for nearby spatially resolved galaxies. For the UV part, all available GALEX observations were used, including the Nearby Galaxy Survey (NGS), Deep (DIS), Medium (MIS), and All Sky Imaging Surveys (AIS). It should be noted that the performance of photometry and astrometry is relatively poor at the edge of the field of view (FOV) of GALEX (Morrissey et al. 2007; Drinkwater et al. 2010), which could potentially affect the UV photometry and astrometric matching with optical data of our sample. However, given a circle FOV of GALEX with a diameter of 1.25 , the area of the edge (annulus with $r>0.575$ ) only covers $15 \%$ of the whole FOV. Therefore, we expect that this edge effect can affect at most $15 \%$ of our sample and it should not change the results significantly.

To select our sample, we require the galaxies to have:

1. spectra classified as "GALAXY"

2. $0.02<z<0.05$

3. stellar mass higher than $10^{9} M_{\odot}$

4. axis ratio greater than 0.7 .

The upper redshift limit is set to ensure that the sample is complete in mass. The lower limit is set to avoid very nearby galaxies which are large in the sky, and for which the sky subtraction and deblending are difficult. The high axis ratio selects face-on galaxies to minimize the effect caused by internal dust extinction. Our final sample consists of 25,074 objects. We use the elliptical Petrosian magnitude from UV to optical in the NSA catalog. ${ }^{3}$ The $K$-corrections and galactic dust extinctions are also taken from the NSA catalog. We do not correct the UV and optical fluxes for internal dust extinction, because such corrections carry large systematical uncertainties and tend to be unreliable. To account for dust without introducing additional uncertainties to the data, we apply dust extinction to stellar population synthesis models to qualitatively describe the extinction suffered by the observed colors. The measurements of the stellar mass were taken from the MPA-JHU catalog (Brinchmann et al. 2004).

\section{HOW FAST DO GALAXIES EVOLVE THROUGH THE TRANSITION ZONE}

\subsection{Stellar Population Synthesis Model}

It is still under debate whether galaxies experience an accelerated SFR decline before turning quiescent. In this work, we investigate both one-phase and two-phase star formation histories (SFHs) based on stellar population synthesis models. For simplicity, we assume that galaxy SFR declines exponentially in each evolution phase. Besides a single exponential decline model as a special case, we generally assume that galaxies experience a two-phase evolution to evolve into the red sequence. In the first star-forming stage, the SFR declines slowly with a long e-folding time. While at some point, the SFR begins to drop rapidly with a shorter e-folding time, which we refer to as the "quenching" stage. In this paper, we differentiate the term describing galaxies going through a particular evolution stage in the model from the term describing galaxies satisfying an observational definition (e.g., a color cut), since there may not be a strict correspondence between the two. In particular, we refer to galaxies going through the second evolution phase as "quenched galaxies." And we refer to galaxies defined by having intermediate colors as "transition galaxies" or "green valley galaxies."

The left panel of Figure 1 illustrates the two-phase SFH definition. There are four parameters in the two-phase SFH: formation time $t_{\mathrm{f}}$, quenching start time $t_{\mathrm{q}}$, initial e-folding time

\footnotetext{
http://www.nsatlas.org/data
} 

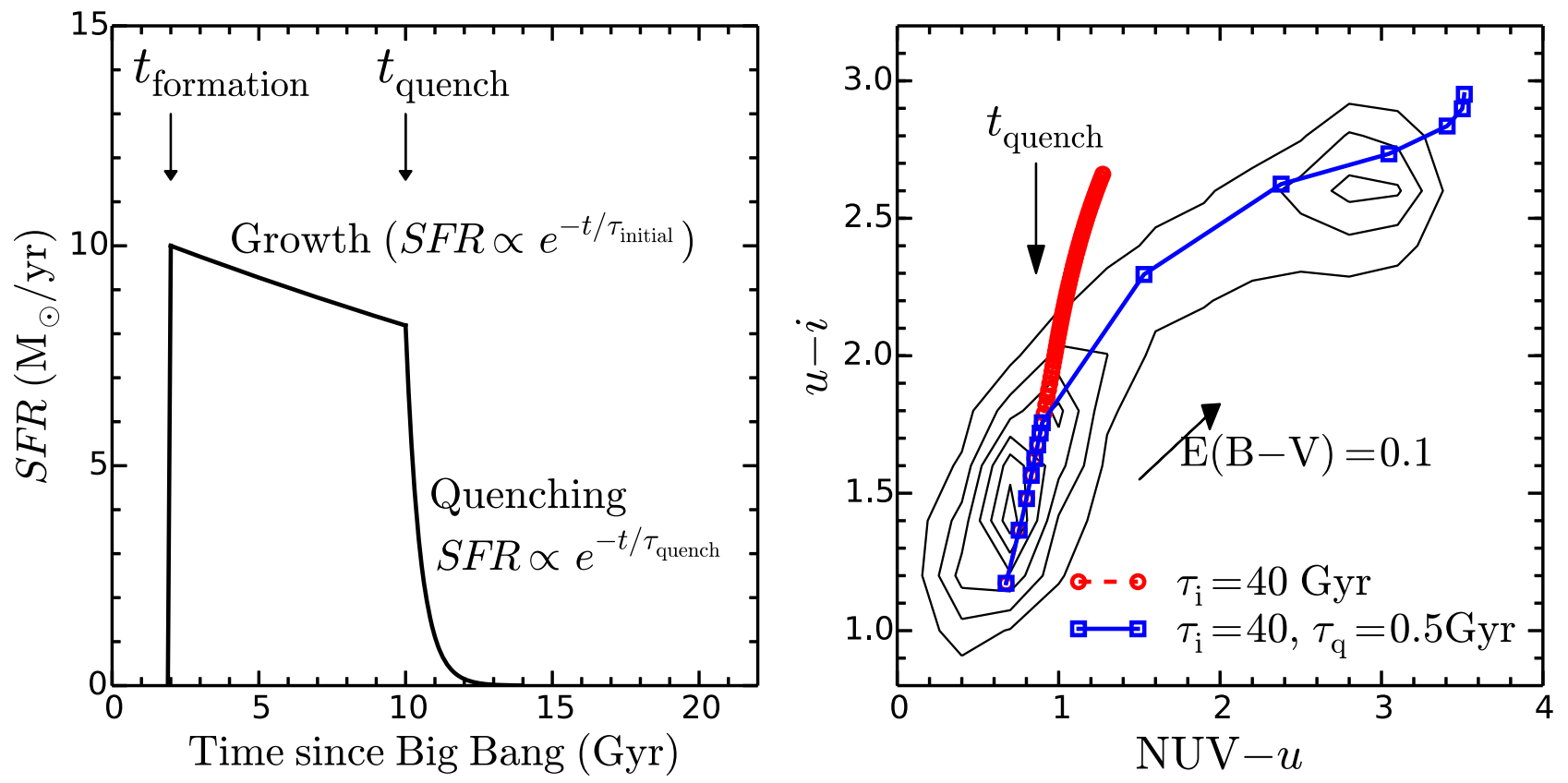

Figure 1. Schematic plot to show the two-phase SFH adopted in the stellar population model. The left panel directly shows the two-phase SFH definition and the four parameters that characterize the SFH. The right panel shows the model applied with the one-phase SFHs (red circles) and two-phase SFH (blue squares) in the NUV $-u$ vs. $u-i$ color-color diagram. The black contour represents the distribution of our galaxy sample. The black arrow indicates the color changing caused by extinction with color excess $E(B-V)=0.1$.

$\tau_{\mathrm{i}}$ for the star-forming stage and e-folding time $\tau_{\mathrm{q}}$ for the quenching stage. The right panel shows the one-phase (red circles) and two-phase (blue squares) models in the NUV $-u$ versus $u-i$ color-color diagram. The black contour represents the distribution of our galaxy sample. It is worth noting that a clear turn of galaxy distribution is present at NUV $-u \sim 1.4$ and a dramatic number density drop can also be seen in between the blue cloud and the red sequence. The symbols on the model track mark the evolution time from 1-14 Gyr with an interval of 1 Gyr. For the one-phase model, we extend the end point to $60 \mathrm{Gyr}$ to illustrate the model's evolution trend. It can be seen that the model track with two-phase SFH successfully reproduces the angled distribution and sparse region in between the main populations. However, the one-phase model, with the e-folding time given by Noeske et al. (2007a), significantly deviates from the angled distribution of the galaxies. Meanwhile, the number density profile of the one-phase model in $\mathrm{NUV}-u$ color space is rather flat, conflicting with the clearly observed low-density region. In other words, the galaxy distribution in the NUV $-u$ versus $u-i$ color-color diagram is a strong evidence for the two-phase evolution scenario.

For the star-forming galaxies, Noeske et al. (2007a) found that the cosmic evolution of the main-sequence relation can be well explained assuming an SFH of single exponential decline. They also derived the e-folding time and formation time as functions of stellar mass in Equation (6) of Noeske et al. (2007a). Generally, more massive galaxies tend to be formed at earlier epochs and their SFR declines more rapidly with a shorter e-folding time. For example, galaxies with masses of $10^{10.1} M_{\odot}, 10^{10.3} M_{\odot}$, and $10^{10.5} M_{\odot}$ formed at redshift $z \sim 1$, 1.5 , and 1.8 with e-folding times of 40,25 , and $16 \mathrm{Gyr}$, respectively. In later analysis, we do not try to fit the properties of galaxies one by one, but only use the models to illustrate the statistical evolutionary trend.
We generate model spectral energy distribution (SED) using the Bruzual \& Charlot (2003) population model spectra with the Chabrier (2003) initial mass function. The metallicity is fixed to the solar value. Following Schawinski et al. (2014) we ignore the contribution from stellar populations younger than $3 \mathrm{Myr}$, because they tend to be surrounded by optically thick clouds and are not visible in the UV and optical. In addition, we apply dust extinction to the model SED with $E$ $(B-V)=0.1$ assuming the Cardelli et al. (1989) extinction law. This amount of extinction is broadly consistent with the estimates by stellar continuum fitting (Oh et al. 2011) for our galaxy sample on average. Since young stellar populations are probably embedded in birth molecular cloud and suffer higher extinction than the old stellar populations, we assume that the stellar populations younger than $10 \mathrm{Myr}$ suffer dust extinction three times higher than the general population. As quiescent galaxies shows negligible dust extinction, we apply a degressive dust extinction to the model after quenching, which drops at a speed proportional to the SFR decline and reaches zero at NUV $-u$ of 3 mag. Finally, we obtain the model colors by convolving the model SED with GALEX and SDSS filter transmission curves.

\subsection{Evolution Speed in the Transition Stage}

Currently, it is still not well understood how fast SFR declines when a galaxy quenches or how fast galaxies evolve through the transition zone. The evolution speed can be characterized by the e-folding time under the assumption of an exponential decline. Generally, the NUV $-u$ color traces the recent star formation activity and is sensitive to the fast SFR decline. On the other hand, the $u-i$ color is more sensitive to older stellar populations and roughly indicates the average stellar age. Therefore, the slope of the transition galaxies in $\mathrm{NUV}-u$ versus $u-i$ color will effectively constrain the e-folding time or evolution speed of the quenching process. 

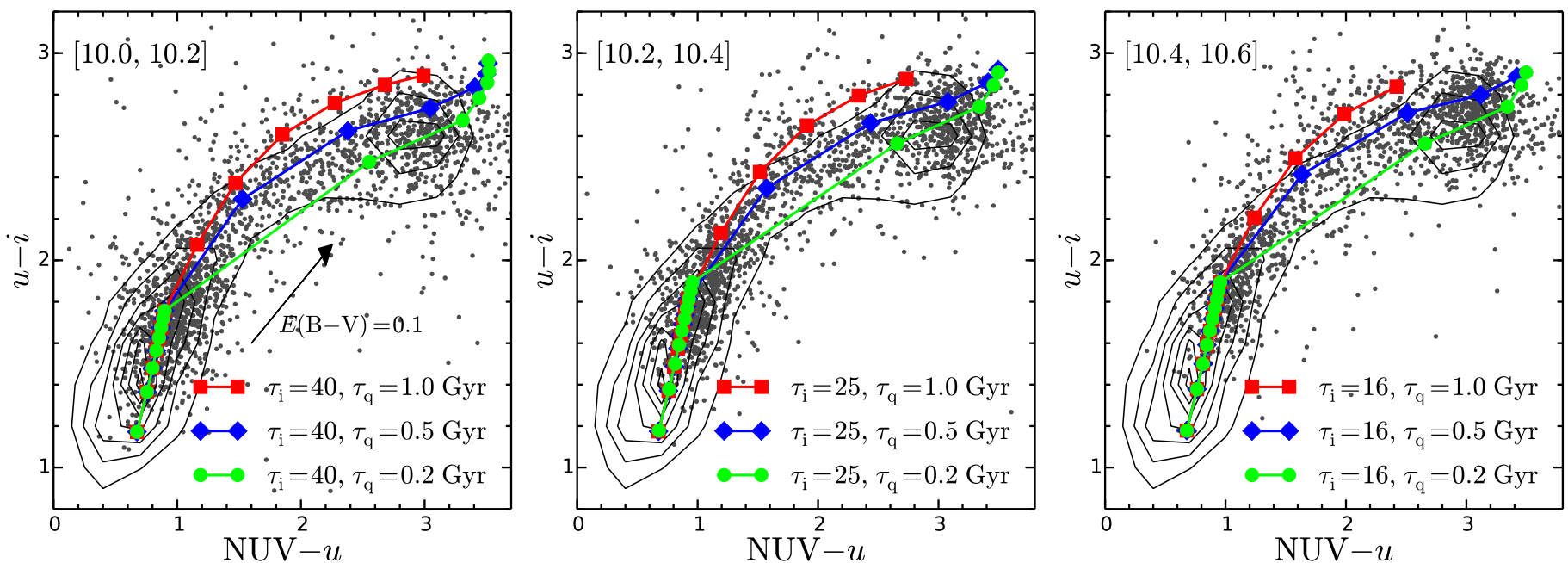

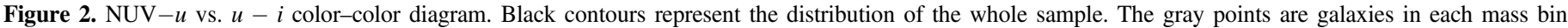

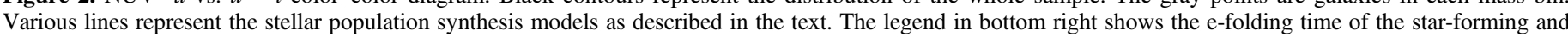
quenching stage for each model track.

Schawinski et al. (2014) provided broad constraint to the e-folding time of transition galaxies with different morphologies using the NUV $-u$ versus $u-i$ diagram. In this work, we investigate the e-folding time of quenching for galaxies split by stellar mass rather than morphologies.

Figure 2 shows the NUV $-u$ versus $u-i$ diagram for the galaxies in three mass bins from $10^{10-} 10^{10.6} M_{\odot}$. The gray points represent the galaxies in each mass bin, while the contours denote the distribution of the whole data sample. The black arrow indicates the effect caused by dust extinction with $E(B-V)=0.1$ Extinction by dust would introduce scatter to the galaxy distribution in the diagonal direction. In each panel, lines are the toy models generated to illustrate the parameter range. The symbols on the model track represent the evolution time from 1-14 Gyr with an interval of $1 \mathrm{Gyr}$. As mentioned above, we adopt the model SFH of two exponential declines with e-folding times of $\tau_{\mathrm{i}}$ and $\tau_{\mathrm{q}}$. The legend in the bottom right shows the value of $\tau_{\mathrm{i}}$ and $\tau_{\mathrm{q}}$ for each model. We adopt the mass-dependent $\tau_{\mathrm{i}}$ given by Noeske et al. (2007a), because it provides a good description of the cosmic evolution of the starforming main-sequence. As we will argue below, galaxies that fall in the green valley must have quenched recently. Thus, for simplicity, we assume their $t_{\mathrm{q}}$ to be the current time. For the three stellar mass bins, their $t_{\mathrm{q}}$ are $8 / 9 / 10 \mathrm{Gyr}$ for the model in the panels from the left to the right, respectively. It can be seen that, in all three mass bins, the model with $\tau_{q}$ of $0.5 \mathrm{Gyr}$ best matches the angled distribution compared to those models with $\tau_{q}$ of 1 and 0.2 Gyr. Models with higher $\tau_{q}$ systematically offset from the data points with redder $u-i$ color, while others with lower $\tau_{q}$ deviate to the opposite direction. Therefore, statistically, the e-folding time of the quenching process should have an average around $0.5 \mathrm{Gyr}$, and will rarely be faster than 0.2 Gyr or longer than $1 \mathrm{Gyr}$.

Our results are broadly consistent with Schawinski et al. (2014), who obtained a wide range of e-folding time for green valleys from $2.5 \mathrm{Gyr}$ for late-type ones to $0.25 \mathrm{Gyr}$ for earlytype ones. The higher e-folding time derived by Schawinski et al. (2014) can be due to their different two-phase SFH which comprises a constant SFR (or infinite e-folding time) in the first 9 Gyr and a subsequent exponential decline. This difference in $\mathrm{SFH}$ results in a different starting point for quenching. As shown in Figure 7, in Schawinski et al. (2014), their model starts quenching at a point much bluer than the main population of star-forming galaxies. This offset is more significant if we limit it to galaxies with $M_{*} \sim 10^{10} M_{\odot}$. Using the SFH given by Noeske et al. (2007a) provides a much more realistic starting point, matching the median color of star-forming galaxies of a given stellar mass. Thus, this approach should provide a better description of the evolution speed during quenching.

\section{QUENCHING FRACTION AND QUENCHING RATE}

\subsection{Number Density Profile}

So far, we have only used the information about the envelope of the galaxies' distribution in color-color space. The number density profile of galaxies in color space is another independent and critical piece of information, which we can utilize to investigate how galaxies likely quench and evolve through the transition zone.

The upper left panel of Figure 3 shows the galaxies in NUV $-u$ colors versus stellar mass diagram, while the other three panels are the number density profile in NUV $-u$ color space for galaxies in three mass bins. In the mass-color diagram, a clear bimodal distribution is present. Interestingly, galaxies in the blue cloud and red sequence are parallel to each other and separated by a wide low-density region, which we refer to as the transition zone hereafter. Blue circles and red squares represent the model tracks, which best match the galaxy distribution in the NUV $-u$ versus $u-i$ color-color diagram for the low- and high-mass bins, respectively. As indicated by the model tracks, galaxy mass barely changes after quenching, while its NUV $-u$ color increases dramatically. This is the basis for our assumption that galaxies with the same mass, but different color, represent different stages of the same evolutionary sequence from star-forming, through transition, and to the red sequence. As discussed above, the spread of starforming galaxies in NUV $-u$ color could probably be mainly due to the distribution of internal dust extinction and small variations in SFR around the long-term average value.

In this work, we focus on galaxies with stellar mass in three narrow bins, from $10^{10-} 10^{10.6} M_{\odot}$. Their number density 

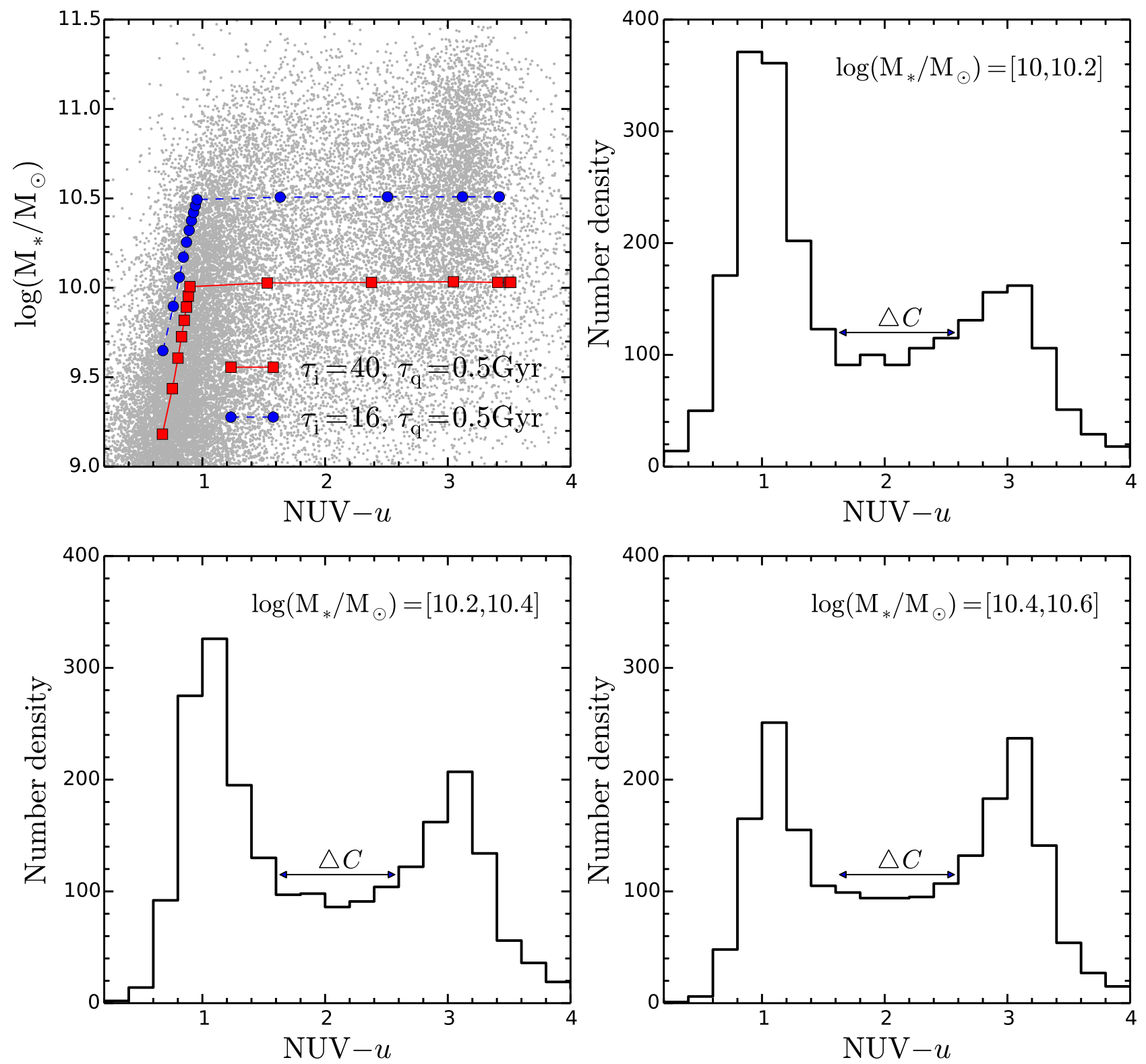

Figure 3. Upper left panel: NUV $-u$ color vs. stellar mass diagram. Blue circles and red squares represent the model tracks, which best represent the galaxy distribution in the NUV $-u$ vs. $u-i$ color-color diagram in the low- and high-mass bin, respectively. Other three panels: Number density profile of galaxies in each mass bin. The color range of the transition zone is marked with $\triangle C$.

profiles are shown in the upper right and bottom panels of Figure 3. It can be seen that the low-density transition zone is clearly separated from the star-forming zone by a dramatic number density drop at NUV $-u$ of $1.4-1.6$. Interestingly, the number densities in the transition zone are roughly the same among the three mass bins with $\sim 100$ objects in each color bin with the width $\Delta C_{\text {bin }}=0.2 \mathrm{mag}$. In the higher-mass bin, there are relatively more passive galaxies than star-forming galaxies, which indicates that the quenching process in high-mass systems has either been happening for a longer period or they evolve faster than low-mass galaxies. According to Noeske et al. (2007a), more massive galaxies formed earlier compared to low-mass systems. It is natural to assume that quenching started earlier among them. In addition, we have shown above that galaxies of different masses have a similar e-folding time during quenching. Therefore, the different ratios between the red sequence and blue cloud at different masses are probably due to different quenching start times.

\subsection{Quenching Rate}

The key to studying the quenching fraction or quenching rate is to answer how the transition zone built up in the past. First, we define the transition zone as the low-density region between star-forming and passive galaxies. As can be seen from the NUV $-u$ versus mass diagram in Figure 3 , the positions of the star-forming galaxies and red-sequence galaxies are nearly constant in NUV $-u$, independent of stellar mass, suggesting that the color range of the transition zone should be similar for the galaxies in the three mass bins. By visually inspecting the number density drop, we define a mass-independent transition zone with NUV $-u$ between 1.6 and 2.6 mag, which is marked 
Table 1

Quenching Fraction for Galaxies of Different Masses (Derived for Transition Zone Defined as $1.6<$ NUV- $u<2.6$ )

\begin{tabular}{lccc}
\hline \hline & $\begin{array}{c}\text { mass bin1 } \\
{[10.0,10.2]}\end{array}$ & $\begin{array}{c}\text { mass bin } 2 \\
{[10.2,10.4]}\end{array}$ & $\begin{array}{c}\text { mass bin 3 } \\
{[10.4,10.6]}\end{array}$ \\
\hline Simple estimation & $28 \%$ & $31 \%$ & $40 \%$ \\
Accurate estimation & $28 \%$ & $35 \%$ & $45 \%$ \\
\hline
\end{tabular}

in Figure 3 as $\triangle C$. We will verify this choice later, when we fit the number density profile.

\subsubsection{A Simple Approach}

We first adopt a simple approach to estimate the fraction of quenching. We assume that all of the galaxies in transition zone are galaxies that quenched during a recent period $T$. We will quantify the period $T$ in the next subsection. As a result, 503/ $476 / 489$ galaxies are found in the transition zone from the lowto high-mass bins, respectively. This similar total number in different mass bins is expected from the number density profile in Figure 3. We define the quenching fraction to be the ratio of the transition galaxies to the sum of the transition galaxies and star-forming galaxies, because we expect that the transition galaxies were part of the star-forming population at a time period $T$ ago. This yields quenching fractions in the three mass bins to be $28 \%, 31 \%$, and $40 \%$, respectively. We include these results in Table 1. It should be noted that these value of the quenching fraction correspond to a time period $T$.

\subsubsection{A More Accurate Approach}

The estimates of the simple method can be inaccurate in two aspects. First, the red end of the transition zone may have contributions from galaxies that quenched more than $T$ Gyr ago. Second, since there is a spread in color among starforming galaxies, the most recently quenched galaxies may still have the same color as other slightly redder star-forming galaxies. For a more accurate estimate of the quenching fraction, we simulate how the star-forming and transition zone built up. It should be noted that there are several basic assumptions adopted in this approach, as follows:

1. At a given stellar mass, star-forming galaxies of different NUV $-u$ colors have the same probability of being quenched.

2. The fraction of quenching is constant over the time interval $T$.

3. Quenched galaxies evolve through the NUV $-u$ color space at a constant speed.

4. Assumptions 1 and 3 above also apply to the time period between $T$ and $2 T$.

We check the third assumption of constant evolution speed using the stellar population models. Figure 4 shows the evolution speed (i.e., color-changing speed) of models in NUV $-u$ color space. The dramatic increase indicates the time when quenching happens. It can be seen that the evolution speed of the models is independent of the initial SFH $\left(\tau_{\mathrm{i}}\right)$, and is roughly constant over the quenching phase. The dashed red line represents the average evolution speed of the model from the beginning to the end of the transition stage. The variation of the evolution speed is less than $30 \%$ and the assumption of a constant value is not far from the truth. In addition, the color-

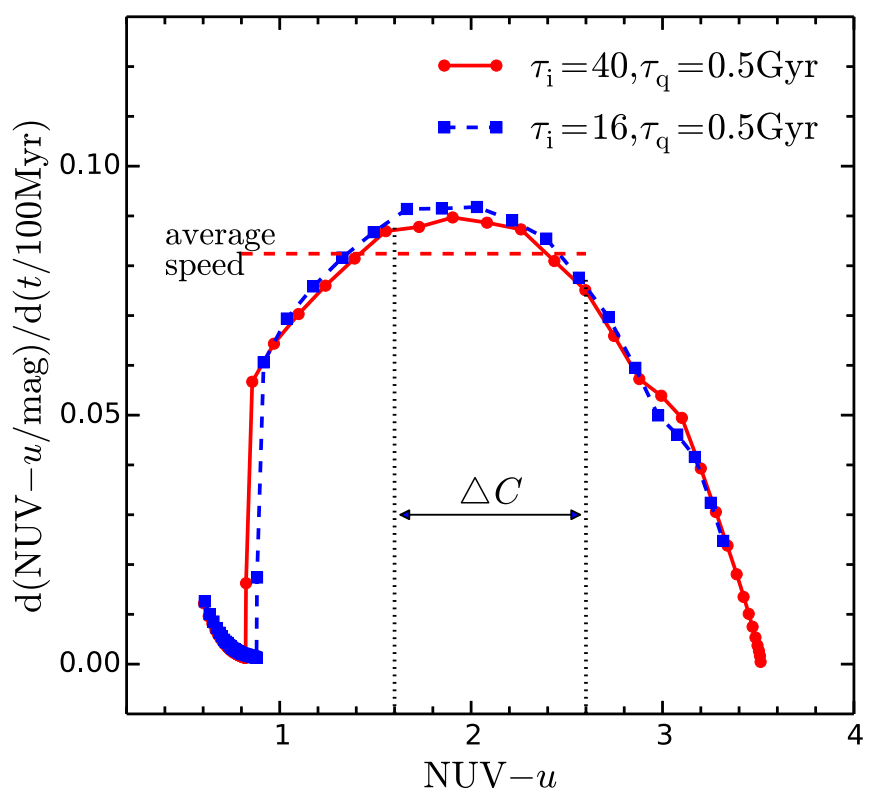

Figure 4. Evolution speed of the models in NUV $-u$ color space. The transition zone is marked as $\Delta \mathrm{C}$. The red dashed line denotes the average evolution speed of the model within the quenching stage.

changing speed in the last several Gyr of the star-forming stage barely changes and is much smaller compared to that during the quenching stage.

Based on the assumption of a constant evolution speed, we could derive the crossing time $T$ of the transition zone. For models with certain $\tau_{i}$ and $\tau_{q}$, we obtain the color-changing speed $v_{i}$ and $v_{q}$. Given that the color range of the transition zone is caused by the differential evolution speed of the two evolution stages, we have

$$
T=\triangle C /\left(v_{2}-v_{1}\right),
$$

where $\triangle C$ is the NUV $-u$ color range of the transition zone. As mentioned above, we adopt $\triangle C$ of 1.0 mag for the three mass bins, as marked in Figure 3. According to our results in Section 3.2, the e-folding time of the quenching stage is generally much shorter than that of the star-forming stage. Therefore, we have $v_{\mathrm{q}} \gg v_{\mathrm{i}}$ and then the time interval $T$ should be determined by the e-folding time of the quenching stage. If we adopt the best-fitting e-folding time of the quenching stage of $0.5 \mathrm{Gyr}$, the time interval $T$ will be $\sim 1.5 \mathrm{Gyr}$.

We run simulations for a number of quenching fractions ranging from $10 \%-60 \%$. For each trial quenching fraction, we simulate the color distribution of the star-forming and transition galaxies based on the above assumptions and by using partial information from the data. We consider galaxies in these color bins to be made up of three components: galaxies which have not yet quenched (star-forming), galaxies quenched during the last $T$ Gyr ("recently quenched") and those which quenched more than $T$ Gyr ago ("older-quenched"). We obtain their numbers and distribution iteratively. At the end, we assess the overall matching of the color distribution using all the color bins.

The simulation procedure can be described in five steps. Step 1: Initially, we assume the star-forming population $T$ Gyr ago is the sum of the galaxies in the current star-forming and the transition zone, and it has the same NUV $-u$ distribution as the present star-forming population (given assumption 1 above). 

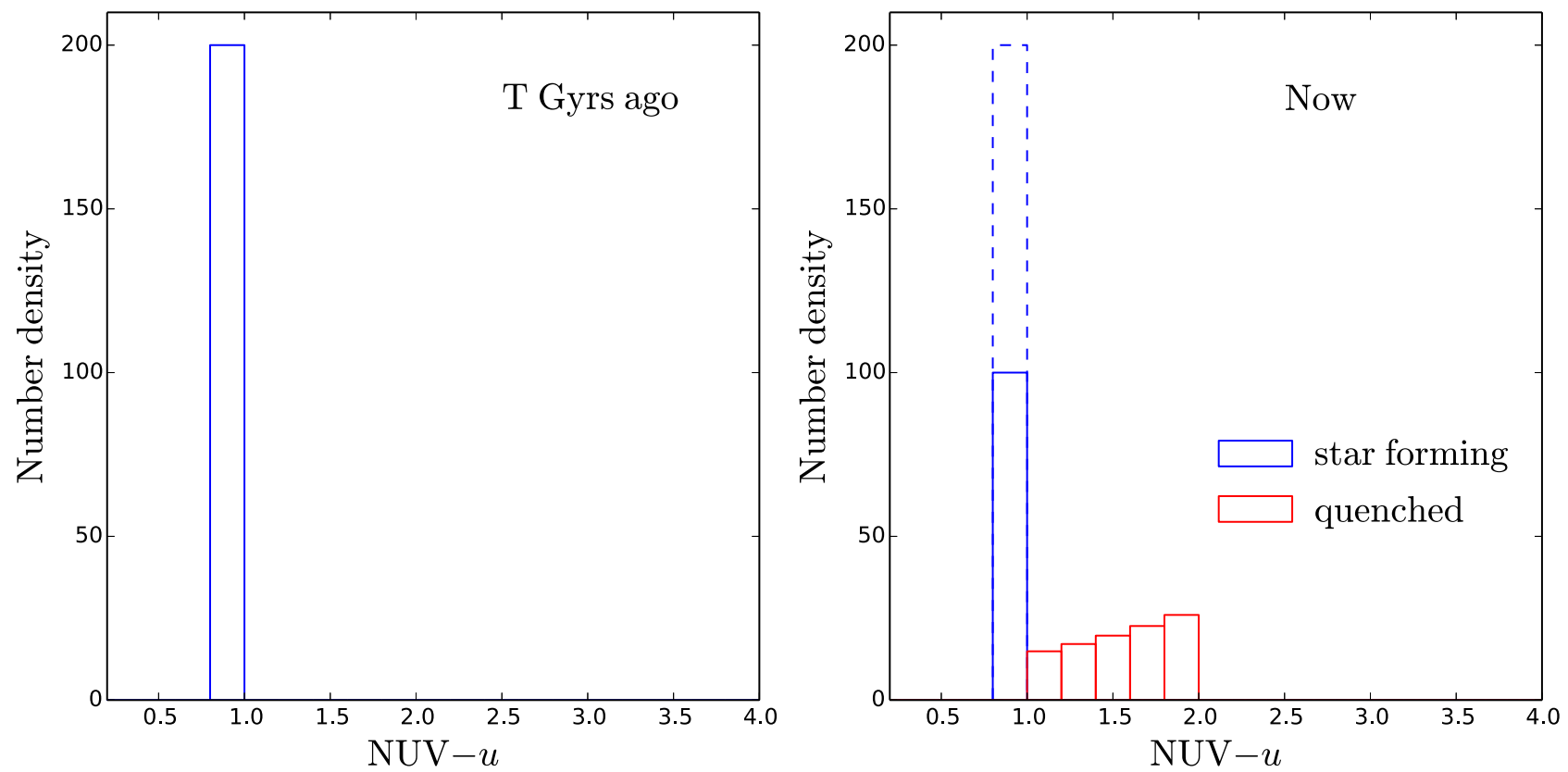

Figure 5. Illustration for the simulation of the quenching process for original star-forming galaxies in one NUV $-u$ color bin from $T$ Gyr, before to the present.

Step 2: For a certain quenching fraction among these original star-forming galaxies, we simulate the quenching process and generate the distribution of the present star-forming population and "recently quenched" population, based on assumptions 2 and 3 above. Figure 5 illustrates the quenching simulation for the original star-forming galaxies in one NUV $-u$ color bin. Each red bar represents the galaxies quenched in each time interval, one fifth of $T$ Gyr. The number of "recently quenched" galaxies declines slightly with time, because the number of star-forming galaxies is declining, which is analogous to the radioactive decay of unstable isotopes.

Step 3: Based on the distribution of the "recently quenched" population, we obtain the distribution of the "older-quenched" population. As implied by the definition of the "olderquenched" population, they are basically analogous to the present "recently quenched" population in the star-forming zone, which will evolve to be the "older-quenched" population in the near future. Therefore, we apply the same distribution of the "recently quenched" population in the star-forming zone to the "older-quenched" population, shifted in color by $\Delta C$ and nomalized so that the "older-quenched" population in the last color bin is equal to the total number in that bin minus the number of the "recently quenched" population.

Now that we have generated an "older-quenched" population, we can refine the total number of the original star-forming population $T$ Gyr ago and its distribution. To update its distribution, we again follow the current distribution of the star-forming population. This needs to exclude the "recently quenched" population in the star-forming zone. Therefore, we use the data and the "older-quenched" population derived above to update the "recently quenched" population.

Step 4: We update the "recently quenched" population in two parts: those in the star-forming zone and those in the transition zone. For the "recently quenched" population in the transition zone $(1.6<\mathrm{NUV}-u<2.6)$, we take the difference between the data and the "older-quenched" population. For those in the star-forming zone (NUV $-u<1.6$ ), we use the same distribution of the previous version, but normalized to the new "recently quenched" population in the first color bin of the transition zone.

Step 5: The new distribution of the star-forming population is then set to be the observed distribution in the star-forming zone, after subtracting the new distribution of the "recently quenched" population. With this new distribution of the starforming population and new number of the "recently quenched" population, we then rerun Steps 2-5 above, until the total number of the star-forming population stops changing.

Although part of the observed color distribution has been used in the iteration above, for each value of the quenching fraction, the simulated distribution (sum of the three components after Step 2) would not necessarily match the data in all the color bins. To assess how the simulated distribution matches the data, we simply calculate the average absolute difference between the simulated distribution and the observed one in each color bin. Finally, we obtain the best-fitting simulated distribution, as shown in Figure 6, with quenching fractions of $28 \%, 35 \%$, and $45 \%$ for the three mass bins from low mass to high mass, respectively. The results of the quenching fraction are summarized in Table 1. These quenching fractions are remarkably consistent with the simple estimates in Section 4.1. Adopting an e-folding time for the quenching stage of $0.5 \mathrm{Gyr}$, which results in a $T$ of $1.5 \mathrm{Gyr}$, we could derive a quenching rate of $19 \% / \mathrm{Gyr}, 25 \% / \mathrm{Gyr}$, and $33 \% /$ Gyr for the three mass bins, respectively.

Considering that the color range of the transition zone is chosen visually according to the number density drop, we also run the simulation above for other color ranges defining the transition zone. For each trial color range, we obtain a best-fit model color distribution. The average difference in the number of galaxies per bin between the model and data provides an indication of the fitting quality. Changing the lower and upper limit of the color range by \pm 0.2 results in much worse fitting, with the number difference per bin much larger than that expected, given Poisson statistics. The only change to the color range, which still results in a reasonable fit, is to decrease the red limit by 0.2 , i.e., defining the transition zone as [1.6, 2.4]. 

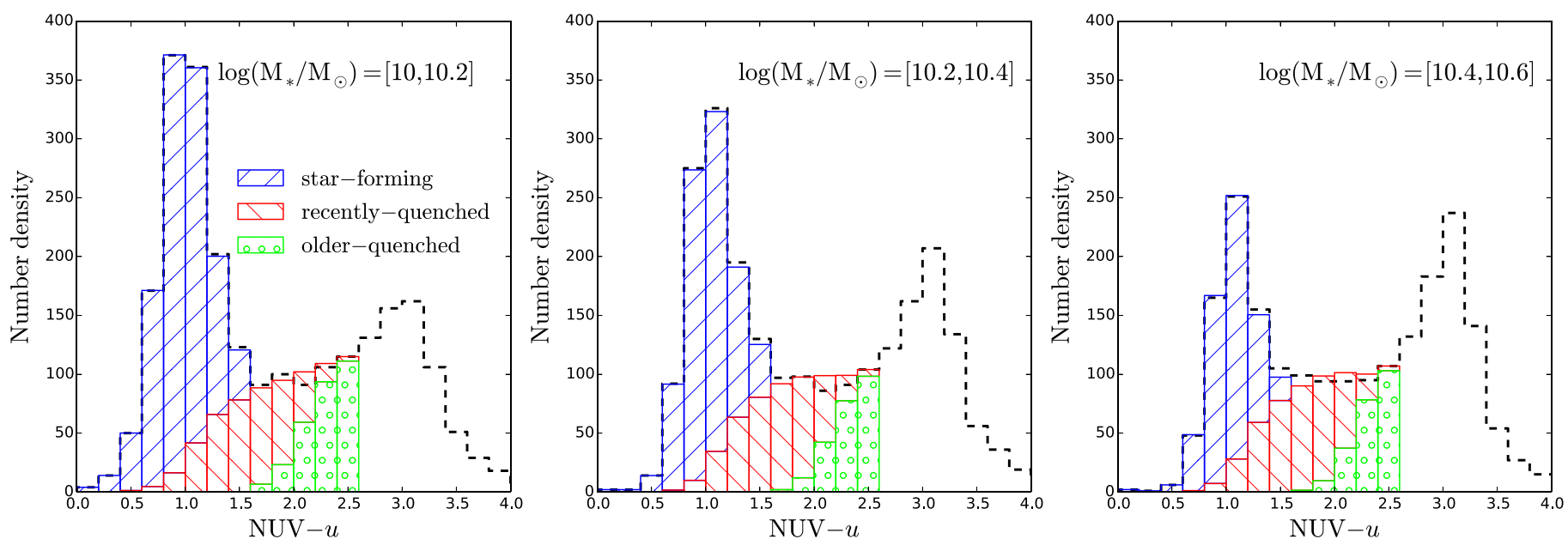

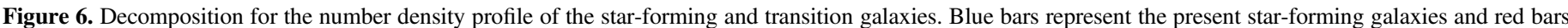

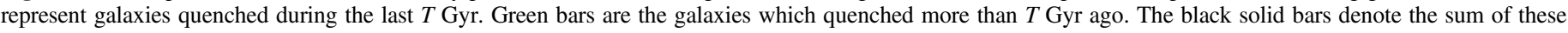
three components. We also show the number density profile of the data sample as a dashed histogram to guide the eye.

In this case, the quenching fraction will be $23 \%, 31 \%$, and $41 \%$ for the three mass bins. Note that the crossing time $T$ will also reduce to 1.2 Gyr. Therefore, the quenching rate will be $19 \% /$ Gyr, 27\%/Gyr, and 36\%/Gyr, which is only slightly higher than the previous one. This confirms the robustness of our result. However, considering the large spread of galaxies around the best-fitting model in the NUV $-u$ versus $u-i$ color-color diagram in Figure 2, notable uncertainties are expected in the e-folding time of the quenching stage and thus in the derived quenching rate.

Moustakas et al. (2013) derived the quenching rate as a function of mass and redshift by investigating the SMF evolution from $z \sim 1-0$. The obtained quenching rate increases with decreasing redshift. However, the dependence of the rate on stellar mass is not monotonic with redshift. At $z \sim 0$, the quenching rate tends to be lower in higher-mass bins, which is the opposite to our expectation. However, at slightly higher redshift, this relationship reverses to a higher quenching rate for higher-mass bins. For star-forming galaxies at low redshift with the stellar mass range adopted in this work, the quenching rate obtained by Moustakas et al. (2013) ranges between $\sim 7 \%-$ $18 \% / \mathrm{Gyr}$, which is systematically lower than our results by a factor of 2 on average. Based on the optical luminosity function, Blanton (2006) found that, from $z \sim 1-0$ ( $~ 8 \mathrm{Gyr}$ ), only $\sim 25 \%$ of star-forming galaxies must have quenched to match the pile-up of passive galaxies. This corresponds to an even lower quenching rate.

There could be several reasons that could account for the discrepancy in the quenching rate obtained in this work and that in the literature. One possible contributor is the passive galaxies with rejuvenated star formation located in the transition zone. These rejuvenated passive galaxies show recent low state or ongoing star formation over the global old stellar population (Fang et al. 2012). Recently, a significant fraction of passive galaxies in the local universe $(\sim 10 \%-30 \%)$ are found to show signatures of star formation in the UV (Yi et al. 2005; Donas et al. 2007; Schawinski et al. 2007) or IR (Shapiro et al. 2010). The origin of these rejuvenated episodes is still under debate and several potential mechanisms are proposed, such as non-axisymmetric structures (e.g., bars), gasrich mergers (e.g., Kaviraj et al. 2009) and gas accretion from an intergalactic medium. Roughly speaking, a rejuvenating rate of $20 \%$ /Gyr, which is typically found in the local universe, can easily account for the discrepancy of the quenching rate in this work and Moustakas et al. (2013). Another reason for the different quenching rate can be the inaccuracy in the e-folding time of the quenching stage. If we adopt a higher e-folding time of $1 \mathrm{Gyr}$ for the quenching stage, the crossing time $T$ will double and thus the quenching rate will shrink by a factor of 2 . However, in the NUV $-u$ versus $u-i$ color-color diagram, the model with e-folding time of $1 \mathrm{Gyr}$ does not represent the majority of galaxies in the transition zone. Some potential inaccuracies in the stellar population model and/or dust extinction could possibly induce a higher e-folding time at the quenching phase, which is comparable with the color-color diagram. We will investigate in detail the reason for the difference in quenching rate in future work.

To date, the mechanisms for triggering quenching are still not well understood. Physical processes that shut down the cold gas supply or speed up the gas depletion rate are needed to cease the star formation in galaxies. Several candidates are proposed, such as strangulation due to shock heating in massive halos, active galactic nucleus (AGN) feedback both in "quasar-mode" triggered by major mergers and "radio-mode" in massive galaxies, bars that drive cold gas into the central region of galaxies where it will be consumed quickly, and rampressure stripping in dense regions (see a detailed discussion in Schawinski et al. 2014 and reference therein). Our quenching rate results should provide important constraints on the proposed quenching processes and their implementation in galaxy evolution models.

\section{SUMMARY}

In this work, we investigated the evolution speed of galaxies in the quenching stage and the quenching fraction of starforming galaxies. We select a local galaxy sample from SDSS with redshift in $[0.02,0.05]$ and target a subsample of them with stellar mass in $\left[10^{10}, 10^{10.6}\right] M_{\odot}$. To reproduce their UVoptical colors, we use stellar population synthesis models. For simplicity, we adopt a model SFH of two-phase exponential decline, which corresponds to the secular star-forming stage and the rapid quenching stage. 
In the NUV $-u$ versus $u-i$ color-color diagram, we find a clear turn along with a dramatic number density drop, which strongly support the two-phase evolution scenario. To constrain the e-folding time of the quenching stage, we compare the model tracks with the transition galaxy distribution in the NUV $-u$ versus $u-i$ color-color diagram. For the quenching stage, the e-folding time should be statistically within $[0.2,1] \mathrm{Gyr}$, while an e-folding time of $0.5 \mathrm{Gyr}$ best matches the observed galaxy distribution. Adopting this best-fitting e-folding time, the crossing time $T$ of the transition zone in NUV $-u$ color is about 1.5 Gyr.

The galaxy number density profile in the NUV $-u$ color space provides critical insight into the quenching fraction during the past period $T$. Using a simple approach and a complicated, but more accurate approach, we have found consistent results for the quenching fraction among starforming galaxies. In the three mass bins, the quenching fractions are found to be $28 \%, 35 \%$, and $45 \%$. Adopting an e-folding time of $0.5 \mathrm{Gyr}$ for the quenching stage, which corresponds to a crossing time $T$ of $1.5 \mathrm{Gyr}$, we further derive a quenching rate of 19\%/Gyr, 25\%/Gyr, and 33\%/Gyr for the star-forming galaxies in the three mass bins. We also examined different definitions of the transition zone and found that our result is insensitive to slight changes of the transition zone definition.

Compared to the quenching rate derived by Moustakas et al. (2013), based on the SMF of star-forming and passive galaxies from $z \sim 1-0$, our results are broadly consistent within the uncertainties, but systematically higher. This discrepancy, to the best of our knowledge, is possibly due to the passive galaxies with rejuvenated star formation in the transition zone and potential inaccuracies in derived e-folding time at the quenching stage. Therefore, our results could be the upper limits of the quenching rate.

We thank Michael Blanton, Jeremy Tinker, and ChangHoon Hahn for helpful conversations about this work. J. Lian gratefully acknowledges support from the China Scholarship Council. This work is supported by the Strategic Priority Research Program "The Emergence of Cosmological Structures" of the Chinese Academy of Sciences (No. XDB09000000), the National Basic Research Program of China (973 Program)(2015CB857004), and the National Natural Science Foundation of China (NSFC, Nos. 11225315, 1320101002, 11433005 and 11421303).

Galaxy Evolution Explorer (GALEX) is a NASA Small Explorer, launched in 2003 April. We gratefully acknowledge NASA's support for the construction, operation and scientific analysis for the GALEX mission, developed in cooperation with the Centre National d'Etudes Spatiales of France and the Korean Ministry of Science and Technology.

Funding for the SDSS and SDSS-II has been provided by the Alfred P. Sloan Foundation, the Participating Institutions, the National Science Foundation, the U.S. Department of Energy, NASA, the Japanese Monbukagakusho, the Max Planck Society, and the Higher Education Funding Council for England. The SDSS Web Site is http://www.sdss.org/.

The SDSS is managed by the Astrophysical Research Consortium for the Participating Institutions. The Participating Institutions are the American Museum of Natural History, the
Astrophysical Institute Potsdam, the University of Basel, the University of Cambridge, the Case Western Reserve University, the University of Chicago, Drexel University, Fermilab, the Institute for Advanced Study, the Japan Participation Group, Johns Hopkins University, the Joint Institute for Nuclear Astrophysics, the Kavli Institute for Particle Astrophysics and Cosmology, the Korean Scientist Group, the Chinese Academy of Sciences (LAMOST), the Los Alamos National Laboratory, the Max-Planck-Institute for Astronomy (MPIA), the Max-Planck-Institute for Astrophysics (MPA), New Mexico State University, Ohio State University, the University of Pittsburgh, the University of Portsmouth, Princeton University, the United States Naval Observatory, and the University of Washington.

\section{REFERENCES}

Baldry, I. K., Glazebrook, K., Brinkmann, J., et al. 2004, ApJ, 600, 681 Balogh, M. L., McGee, S. L., Wilman, D. J., et al. 2011, MNRAS, 412, 2303 Bell, E. F., Wolf, C., Meisenheimer, K., et al. 2004, ApJ, 608, 752 Blanton, M. R. 2006, ApJ, 648, 268

Blanton, M. R., Kazin, E., Muna, D., Weaver, B. A., \& Price-Whelan, A. 2011, AJ, 142, 31

Brammer, G. B., Whitaker, K. E., van Dokkum, P. G., et al. 2009, ApJL, 706, L173

Brinchmann, J., Charlot, S., White, S. D. M., et al. 2004, MNRAS, 351, 1151 Brown, M. J. I., Dey, A., Jannuzi, B. T., et al. 2007, ApJ, 654, 858 Bruzual, G., \& Charlot, S. 2003, MNRAS, 344, 1000

Cardelli, J. A., Clayton, G. C., \& Mathis, J. S. 1989, ApJ, 345, 245 Chabrier, G. 2003, PASP, 115, 763

Daddi, E., Dickinson, M., Morrison, G., et al. 2007, ApJ, 670, 156

Donas, J., Deharveng, J.-M., Rich, R. M., et al. 2007, ApJS, 173, 597

Drinkwater, M. J., Jurek, R. J., Blake, C., et al. 2010, MNRAS, 401, 1429

Driver, S. P., Allen, P. D., Graham, A. W., et al. 2006, MNRAS, 368, 414

Elbaz, D., Daddi, E., Le Borgne, D., et al. 2007, A\&A, 468, 33

Faber, S. M., Willmer, C. N. A., Wolf, C., et al. 2007, ApJ, 665, 265

Fang, J. J., Faber, S. M., Salim, S., Graves, G. J., \& Rich, R. M. 2012, ApJ, 761,23

Gonçalves, T. S., Martin, D. C., Menéndez-Delmestre, K., Wyder, T. K., \& Koekemoer, A. 2012, ApJ, 759, 67

Kauffmann, G., Heckman, T. M., Tremonti, C., et al. 2003, MNRAS, 346, 1055

Kaviraj, S., Peirani, S., Khochfar, S., Silk, J., \& Kay, S. 2009, MNRAS, 394, 1713

Madau, P., \& Dickinson, M. 2014, ARA\&A, 52, 415

Martin, D. C., Wyder, T. K., Schiminovich, D., et al. 2007, ApJS, 173, 342

Martin, D. C., Fanson, J., Schiminovich, D., et al. 2005, ApJL, 619, L1

Mendez, A. J., Coil, A. L., Lotz, J., et al. 2011, ApJ, 736, 110

Morrissey, P., Conrow, T., Barlow, T. A., et al. 2007, ApJS, 173, 682

Moustakas, J., Coil, A. L., Aird, J., et al. 2013, ApJ, 767, 50

Noeske, K. G., Faber, S. M., Weiner, B. J., et al. 2007a, ApJL, 660, L47

Noeske, K. G., Weiner, B. J., Faber, S. M., et al. 2007b, ApJL, 660, L43

Oh, K., Sarzi, M., Schawinski, K., \& Yi, S. K. 2011, ApJS, 195, 13

Pan, Z., Kong, X., \& Fan, L. 2013, ApJ, 776, 14

Ramos, B. H. F., Pellegrini, P. S., Benoist, C., et al. 2011, AJ, 142, 41

Salim, S., Dickinson, M., Michael Rich, R., et al. 2009, ApJ, 700, 161

Schawinski, K., Kaviraj, S., Khochfar, S., et al. 2007, ApJS, 173, 512

Schawinski, K., Urry, C. M., Simmons, B. D., et al. 2014, MNRAS, 440, 889

Shapiro, K. L., Falcón-Barroso, J., van de Ven, G., et al. 2010, MNRAS, 402, 2140

Smethurst, R. J., Lintott, C. J., Simmons, B. D., et al. 2015, MNRAS, 450, 435 Strateva, I., Ivezić, Ž., Knapp, G. R., et al. 2001, AJ, 122, 1861

Trayford, J. W., Theuns, T., Bower, R. G., et al. 2016, MNRAS, 460, 3925 Wetzel, A. R., Tinker, J. L., \& Conroy, C. 2012, MNRAS, 424, 232

Wetzel, A. R., Tinker, J. L., Conroy, C., \& van den Bosch, F. C. 2013, MNRAS, 432, 336

Wong, O. I., Schawinski, K., Kaviraj, S., et al. 2012, MNRAS, 420, 1684 Yang, Y., Zabludoff, A. I., Zaritsky, D., \& Mihos, J. C. 2008, ApJ, 688, 945 Yi, S. K., Yoon, S.-J., Kaviraj, S., et al. 2005, ApJL, 619, L111 